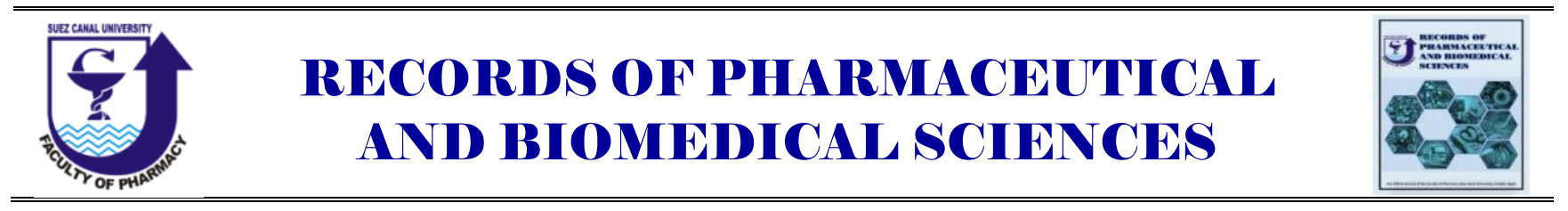

\title{
Cardiotoxicity of Naja nubiae snakebites: A review and consideration
}

\author{
Mohammed Elsayed $^{\text {a* }}$, Mona F. El-Azab ${ }^{a}$, Yasser M. Moustafa ${ }^{a}$, Tarek R. Rahmy ${ }^{b}$ \\ ${ }^{a}$ Department of Pharmacology and Toxicology, Faculty of Pharmacy, Suez Canal University, Ismailia 41522, \\ Egypt; ${ }^{b}$ Department of Zoology, Faculty of Science, Suez Canal University, Ismailia 41522, Egypt
}

Received on: 20. 10. 2020

Revised on: 25. 12. 2020

Accepted on: 29.12. 2020

*Correspondence Author:

Tel: 066/3668284

E-mail address:

mohamed.elsayed@pharm.suez.edu.eg

\begin{abstract}
Snakebite is a worldwide health problem that threats the life of the human being. Although the majority of snake species are non-venomous and typically kill their prey with constriction rather than venom, venomous snakes can be found on every continent except Antarctica. Venomous snakes belong to five families: Elapidae, Crotalidae, Viperidae, Hydrophidae and Colubridae, and are capable of producing venom which is used primarily for immobilizing prey and defense mostly via mechanical injection by special fangs. The venoms contain a vast number of substances with different biochemical and pharmacological activities. Cardiotoxins (CTXs), a group of major venom polypeptides of around 60 amino acid residues present abundantly in the elapid snakes, show pharmacological functions including hemolysis, cytotoxicity and depolarization of muscles. Cardiotoxins are the most abundant toxin components of the venoms of cobra snakes. Additionally, cobra cardiotoxin induce severe tissue necrosis and systolic heart arrest in snakebite.
\end{abstract}

Keywords: Snakebite; Cardiotoxins; Snake venoms.

\section{Introduction}

Snakebite is a worldwide health problem that threats the life of the human being. Although the majority of snake species are non-venomous and typically kill their prey with constriction rather than venom, venomous snakes can be found on every continent except Antarctica (Kasturiratne $\boldsymbol{e t}$ al., 2008). Venomous snakes belong to five families: Elapidae, Crotalidae, Viperidae, Hydrophidae and Colubridae, and are capable of producing venom which is used primarily for immobilizing prey and defense mostly via mechanical injection by special fangs. The venoms contain a vast number of substances with different biochemical and pharmacological activities (Tambourgi et al., 1994). The Nubian spitting cobra is a new species that for long time was considered to be subspecies of Naja pallida, but it was separated due to morphological, through its throat and neck pattern. In envenoming, bites from the Naja nubiae species are commonly associated with local tissue damages, which could be attributed to the svPLA2 and cytotoxins present in these venoms (Petras et al., 2011). Previous studies also indicated that cobra SVPLA2 enzymes worked 
synergistically in case of African spitting cobras with cytotoxins (cardiotoxins) to enhance venom toxicity (Bougis et al.; Tan and Armugam, 1990). Snake venoms are secretion of venomous snake which are synthesized and which are stored in venomous gland. The glands which secrete the zootoxin is a modification of the parotid salivary gland and are situated on each side of head below and behind the eye encapsulated in muscular sheath. Snake venom is a combination of many different proteins, peptides and enzymes and they are generally not dangerous when ingested. Snake venoms are complex mixture of enzymatic and toxic proteins, which include phospholipase A2 (Leon et al., 2011),

Cardiotoxins (CTXs), a group of major venom polypeptides of around 60 amino acid residues present abundantly in the elapid snakes, show pharmacological functions including hemolysis, cytotoxicity and depolarization of muscles (Dufton and Hider, 1988). Cardiotoxins are the most abundant toxin components of the venoms of cobra snakes (Jeyaseelan et al., 1998). Wang et al. added that cobra cardiotoxin induce severe tissue necrosis and systolic heart arrest in snakebite victims (Wang et al., 2006).

\section{Review of literature}

Cobra venom cardiotoxins (CVCs), a PLA2s), myotoxins, hemorrhagic metalloproteinases and other proteolytic enzymes, coagulant components, neurotoxins, cytotoxins and cardiotoxins (Kini, 2003; lso known as cardiotoxins, are basic amphipathic proteins that contain a rigid spatial structure, including anti-parallel $\beta$-sheets (stabilized by four conserved disulfide bonds) and three elongated finger-like loops, each with polar tips that are flanked by small stretches of highly conserved positively charged Lys and Arg residues (figure 1) (Gasanov, 2014). In addition, cardiotoxins have been classified as either S-type cytotoxins (contain Ser in residue position 28 of loop II) or as P-type cytotoxins (contain Pro in residue position 30 of loop II) (figure 1) (Dubovskii et al., 2005). The molecular mechanisms by which CVCs promote cellular pathology remain unclear. Some evidence has shown that cytotoxins can target the membranes of intracellular organelles, such as lysosomes (Wu et al., 2013), and mitochondria (C.-H. Wang and Wu, 2005). For instance, Naja cardiotoxin 3 (CTX3) has been shown to target mitochondria to induce oxidative stress, leading to a collapse of the mitochondrial transmembrane potential, release of cytochrome $\mathrm{C}$, and activation of apoptosis (Chen et al., 2008).

Myocardial infarction (MI), also known as "heart attack," is the death of cardiac muscle resulting from ischemia. It is by far the most important form of ischemic heart diseases and alone is the leading cause of death in the United States and industrialized nations. About 1.5 million individuals in the United States suffer an acute MI annually and approximately one third of them die. At least 250,000 people a year die of a heart attack before they reach the hospital (Ashley \& Niebauer, 2004).

Lipid peroxidation and excessive production of reactive oxygen species (ROS), such as superoxide anions $\left(\mathrm{O}^{2 \cdot-}\right)$ and hydrogen peroxide $\left(\mathrm{H}_{2} \mathrm{O}_{2}\right)$, play a major role in the pathogenesis of MI during oxygen shortage. ROS directly injure the cell membrane and cause cell death. ROS also stimulate signal transduction to upregulate inflammatory cytokines, e.g. tumor necrosis factor- $\alpha(\mathrm{TNF}-\alpha)$ in the ischemic region and surrounding myocardium. (Miyao et al., 1999)

Myocardial infarction (MI) can be recognized by clinical features, including elevated values of biochemical markers (such as cTn or the MB fraction of creatine kinase (CK-MB), electrocardiographic (ECG) findings, and by imaging ( Thygesen et al., 2012).

Myocardial cell death can be recognized by the appearance in the blood of different proteins released into the circulation from the damaged myocytes including myoglobin, cardiac troponin $\mathrm{T}$ and I, CK, CK-MB, LDH, as well as many others. Myocardial infarction is diagnosed when blood levels of sensitive and specific biomarkers such as cardiac troponin or CK-MB are increased in the clinical setting of acute myocardial ischemia. Troponins and CK-MB appear in blood within 6 hrs of infarction, (Tn stay 10 days, CK-MB returns within 48 hours (Figure 2) (Miller et al., 2004).

Nearly three-fourths of patients have one or more complications following acute MI, which include the following (Jones et al., 2011):

A- Contractile dysfunction: Myocardial infarcts produce abnormalities in left ventricular function approximately proportional to their size. Most often, there is some degree of left ventricular 
failure with hypotension, which may progress to pulmonary edema with respiratory impairment. Severe "pump failure" (cardiogenic shock) occurs in $10 \%$ to $15 \%$ of patients following acute MI, generally with a large infarct (often greater than $40 \%$ of the left ventricle).

B- Arrhythmias: Many patients have conduction disturbances and myocardial irritability following MI, which undoubtedly are responsible for many of the sudden deaths.

C- Myocardial rupture: The cardiac rupture syndromes result from the mechanical weakening that occurs in necrotic and subsequently inflamed myocardium and include (1) rupture of the ventricular free wall (most commonly), (2) rupture of the ventricular septum (less commonly), leading to a left-to-right shunt and (3) papillary muscle rupture (least commonly).

Myocarditis is an inflammatory disease of the muscular tissues of heart that may present with sudden cardiac death, symptoms resembling myocardial infarction, heart rhythm and conduction disorders, and heart failure. Infectious, mostly viral, auto-immune and toxic agents are the main etiologies for the pathogenesis of myocarditis. The incidence of myocarditis is largely unknown because of difficulties establishing the diagnosis and the lack of a gold standard other than myocardial biopsy (Maron et al., 2009). Snake bites have been -among other factors- implicated in inducing an autoimmune reaction in the heart that is often characterized by acute rash, fever, peripheral eosinophilia, and ECG abnormalities such as nonspecific ST segment changes or infarct patterns. However, some patients present with sudden death or rapidly progressive Heart Failure. The true incidence is unknown, but in some series, the prevalence of clinically undetected HSM in explanted hearts ranged from 2.4 to 7 percent (Sheikh et al. 2018). Myocarditis of this type is usually associated with hyper eosinophilia that it may be called eosinophilic myocarditis (EM). Endomyocardial biopsy is considered to be a gold standard for the diagnosis of EM. Echocardiography, cardiac magnetic resonance, and bio markers particularly serum eosinophilic cationic protein concentrations are also known to aid in diagnosis. EM may lead to progressive, irreversible, and fatal myocardial damage if prompt diagnosis is not made and therapy is not initiated. The current treatment regimens include corticosteroids, cytotoxic agents, and immunosuppressive therapy. However, a proper treatment criterion is yet to be established (Kindermann et al. 2012).

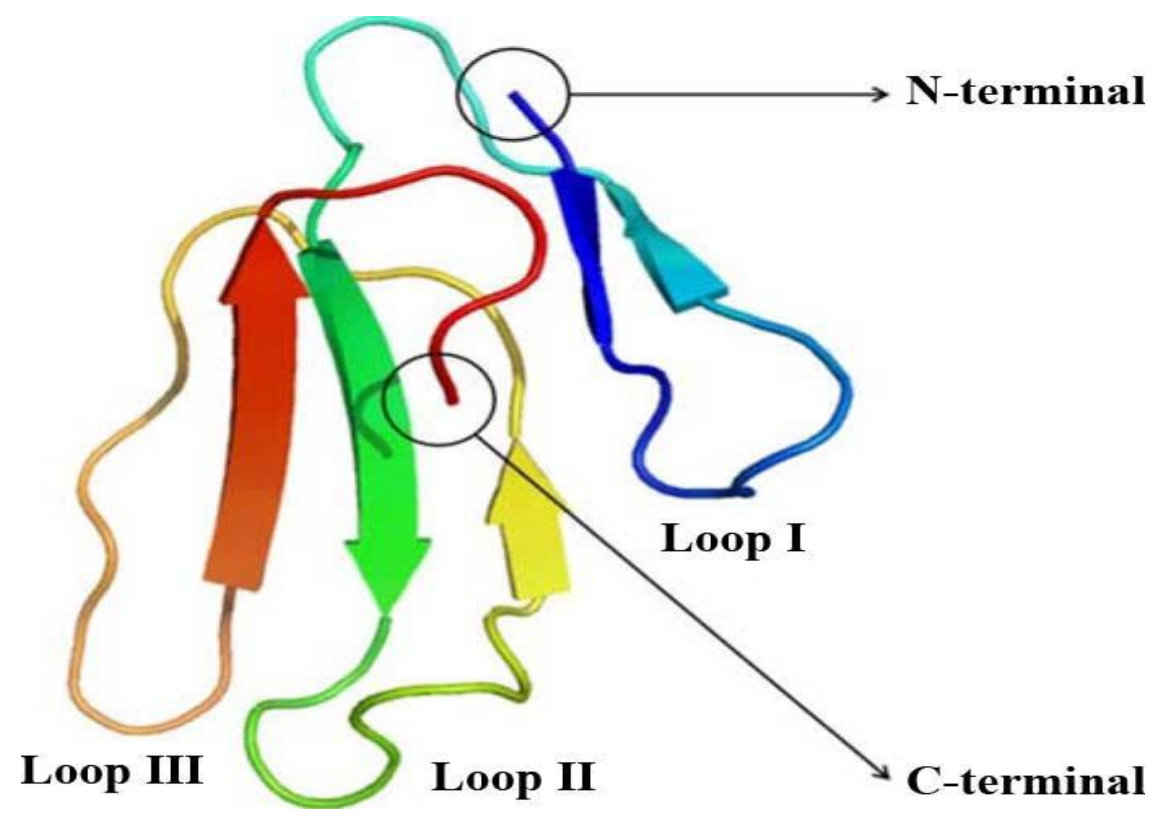

Figure 1: Structure of the cobra cardiotoxin (CTX) protein 


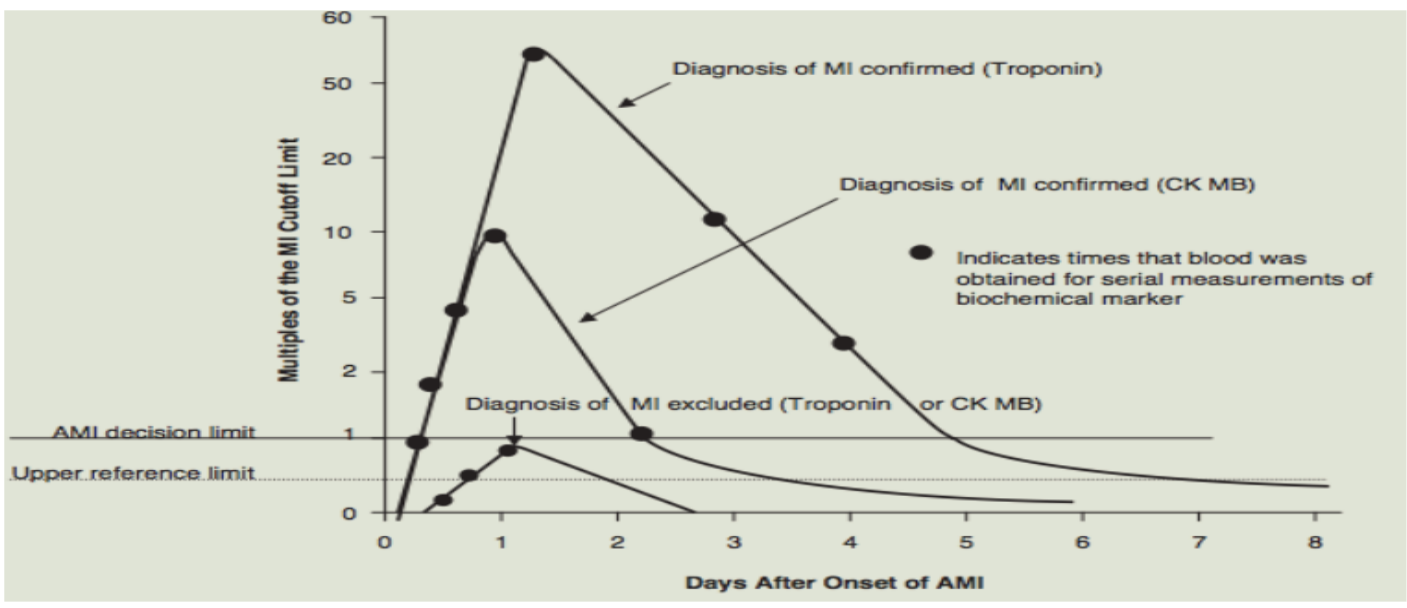

Figure 2: Changes in cardiac markers with time in AMI

The gold standard in diagnosis of myocarditis is still the myocardial biopsy. Acute myocarditis is defined by lymphocytic infiltrates in association with myocyte necrosis. While borderline myocarditis is characterized by inflammatory infiltrates without evidence of myocyte necrosis (Aretz., 1987). This technique is limited by the high variability in interpreting biopsy specimens (in particular with regard to borderline myocarditis) and because noncellular inflammatory processes cannot be detected. Thus, immunohistochemistry is gaining further acceptance in the diagnosis of myocarditis. Monoclonal antibodies allow the characterization and localization of the mononuclear cell infiltrates (Pawlak and Gil, 2017).

Autopsy results of myocardial tissues with myocarditis usually demonstrate increased cardiac weight, intramyocardial extravasation of blood, and fibrin or fibrin-platelet microthrombi in capillaries. Features in keeping with myocardial necrosis on electron microscopy include hypercontraction bands, myofibrillar damage, lysis, intramitochondrial electron dense inclusions, and fibrin deposition in myocyte cytoplasm.

Several subsequent gross pathological examinations have confirmed that myocardial edema, thickening of the left ventricular wall and interventricular septum, myocardial necrosis, pericardial effusions, and fibrinous pericarditis may present in such cases which subsequently lead to severe cardiac toxicity and dilated cardiomyopathies as can be seen in figure 3 (Yeh et al. 2004).

\section{Future recommendation:}

Thymoquinone, a bioactive molecule with wide range of promising effects is known for being both antioxidant and cardioprotective in diverse studies in literature. Studying its protective effect against snake crude venom and elucidating a possible protective pathway could open a new way in the process of alleviating Naja nubiae known cardiotoxicity. It was demonstrated that TQ possessed promising cardioprotective effects in isoproterenol-induced myocardial injury in rats. Isoproterenol is known to induce myocardial lesions similar to acute myocardial infarction in rats. TQ treatment augmented anti-oxidant activity and salvaged cardiomyocytes evidenced by restoration of cardiac injury enzymes and reduction of lipid peroxidation product and pro-inflammatory cytokines. The findings were further supported by histopathological preservation of the myocardium as evidenced by reduced myonecrosis, edema, and infiltration of inflammatory cells. It was revealed that TQ exert protective effect on cardiac injury by attenuating oxidative stress, enhancing endogenous anti-oxidant $\mathrm{s}$ and maintaining structural integrity (Ojha et al., 2015). Thymoquinone supplementation could take part in the antivenin treatment regimens to protect against venominduced cardiotoxicity in individuals living near or where Naja nubiae species inhabit in areas of upper Egypt. In this context, people living in those area can be encouraged to cultivate and implement proportions of black cumin seeds in their food regimes benefiting from TQ discovered effects of cardioprotection. 


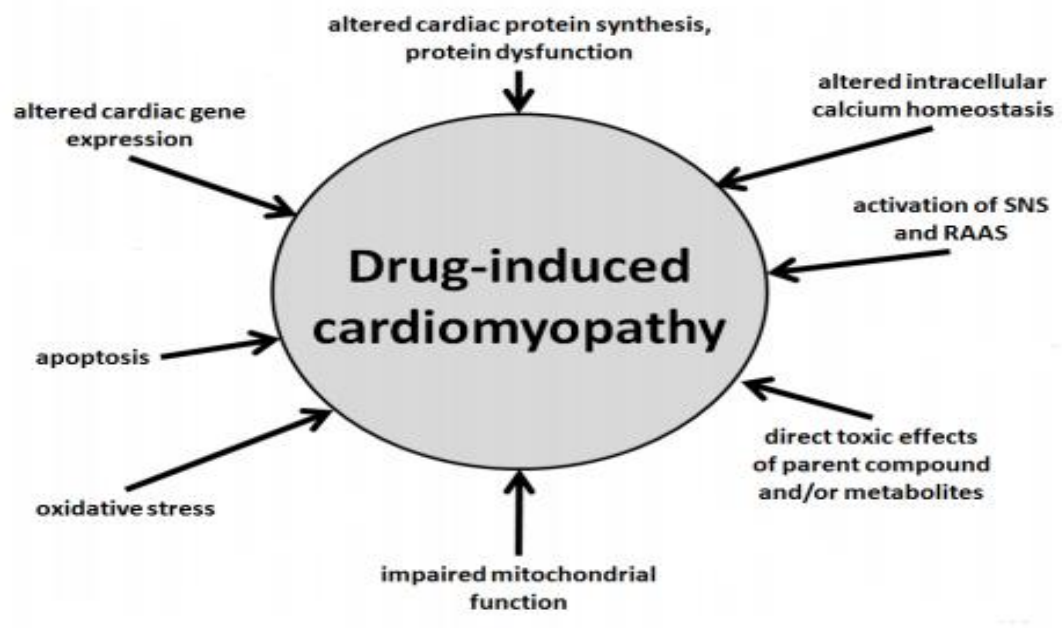

Figure 3: Different pathogenesis of myocarditis and subsequent DCM

\section{References}

Aretz, H.T.; Billingham, M.E.; Edwards, W.D.; Factor, S.M.; Fallon, J.T.; Fenoglio, J.J.; Olsen, E.G. and Schoen, F.J. (1987): "Myocarditis. A Histopathologic Definition and Classification". The American Journal of Cardiovascular Pathology, 1 (1): 3-14.

Ashley, E.A. and Niebauer, J. (2004): "Cardiology Explained", London.

Bougis, P.E.; Marchot, P. and Rochat, H. (1987): "In Vivo Synergy of Cardiotoxin and Phospholipase A2 from the Elapid Snake Naja Mossambica Mossambica". Toxicon, 25 (4): 427-31.

Chang, J.M.; Tseng, F.G. and Chieng, C.C. (2010): "Mixed-SAM Surfaces Monitoring CTX-Protein Part I: Using Atomic Force Microscope Measurements." IEEE Transactions on NanoBioscience, 9 (4): 289-96.

Chen, K.C.; Chiou, Y.L.; Kao, P.H.; Lin, S.R. and Chang, L.S. (2008): "Taiwan Cobra Cardiotoxins Induce Apoptotic Death of Human Neuroblastoma SK-N-SH Cells Mediated by Reactive Oxygen Species Generation and Mitochondrial Depolarization". Toxicon, 51 (4): 624-34.

Dubovskii, P.V.; Lesovoy, D.M.; Dubinnyi, M.A.; Konshina, A.G.; Utkin, Y.N.; Efremov, R.G. and Arseniev, A.S. (2005): "Interaction of Three-Finger
Toxins with Phospholipid Membranes: Comparison of S- and P-Type Cytotoxins". Biochemical Journal, 387 (3): 807-15.

Dufton, M. J. and Hider, R. C. (1988): "Structure and pharmacology of elapid cytotoxins". Pharmacology \& Therapeutics, 36(1): 1-40.

Gasanov, S.E. 2014. "Snake Venom Cytotoxins, Phospholipase A2s, and Zn2+-Dependent Metalloproteinases: Mechanisms of Action and Pharmacological Relevance". Journal of Clinical Toxicology, 4 (1).

Jeyaseelan, K.; Armugam, A.; Lachumanan, R.; Tan, C. H. and Tan, N. H. (1998): "Six isoforms of cardiotoxin in malayan spitting cobra (Naja naja sputatrix) venom: cloning and characterization of cDNAs". Biochimica et Biophysica Acta, 1380(2): 209-222.

Jones, A.; Abramson, S. and Romanelli, J. (2011): "Simultaneous rupture of ventricular septum and papillary muscle as a mechanical complication of acute myocardial infarction". Journal of the American College of Cardiology, 57: e13.

Kasturiratne, A.; Wickremasinghe, A.R.; De Silva, N.; Gunawardena, N.K.; Pathmeswaran, A.; Premaratna, R.; Savioli, L.; Lalloo, D.G. and De Silva, H.J. (2008): "The Global Burden of Snakebite: A Literature Analysis and Modelling Based on Regional Estimates of Envenoming and 
Deaths". Edited by Ken Winkel. PLoS Medicine, 5 (11): e218.

Kindermann, I.; Barth, C.; Mahfoud, F.; Ukena, C.; Lenski, M.; Yilmaz, A.; Klingel, K.; Kandolf, R.; Sechtem, U.; Cooper, L.T. and Böhm, M. (2012): "Update on Myocarditis". Journal of the American College of Cardiology, 59 (9): 779-92.

Kini, R.M. (2003): "Excitement ahead: structure, function and mechanism of snake venom phospholipase A2 enzymes". Toxicon, 42(8): 827840.

Klimas, J. (2012): "Drug-Induced Cardiomyopathies". Cardiomyopathies - From Basic Research to Clinical Management, edited by Josef Veselka. InTech.

Leon, G.; Sanchez, L.; Hernandez, A.; Villalta, M.; Herrera, M.; Segura, A.; Estrada, R. and Gutierrez, J. M. (2011): "Immune response towards snake venoms". Inflammatory Allergy Drug Targets, 10(5): 381-398.

Maron, B.J.; Doerer, J.J.; Haas, T.S.; Tierney, D.M. and Mueller, F.O. (2009): "Sudden Deaths in Young Competitive Athletes: Analysis of 1866 Deaths in the United States, 1980-2006". Circulation, 119 (8): 1085-92.

Miller, W.L.; Garratt, K.N.; Burritt, M.F.; Reeder, G.S. and Jaffe, A.S. (2004): "Timing of peak troponin $\mathrm{T}$ and creatine kinase-MB elevations after percutaneous coronary intervention". Chest, 125(1): 275-280.

Miyao, Y.; Miyazaki, S.; Goto, Y.; Itoh, A.; Daikoku, S.; Morii, I.; Matsumoto, T. and Nonogi, H. (1999): "Role of cytokines and adhesion molecules in ischemia and reperfusion in patients with acute myocardial infarction". Japanese circulation journal, 63: 362-366.

Ojha, S.; Azimullah, S; Mohanraj, R.; Sharma, S.; Yasin, J.; Arya, D.S. and Adem, A. (2015): "Thymoquinone Protects against Myocardial Ischemic Injury by Mitigating Oxidative Stress and Inflammation". Evidence-Based Complementary and Alternative Medicine, 2015: 1-12.

Pawlak, A. and Gil, R.J. (2017): "Infective Cardiomyopathy." Cardiomyopathies - Types and Treatments, edited by Kaan Kirali. InTech.

Petras, D.; Sanz, L.; Segura, Á.; Herrera, M.;
Villalta, M.; Solano, D.; Vargas, M.; Leon, G.; M.; Warrell, D.A.; Theakston, R.D.G.; Harrison, R.A.; Durfa, N.; Nasidi, A.; Gutiérrez,J.M. and Calvete, J.J. (2011): "Snake Venomics of African Spitting Cobras: Toxin Composition and Assessment of Congeneric Cross-Reactivity of the Pan-African EchiTAb-Plus-ICP Antivenom by Antivenomics and Neutralization Approaches". Journal of Proteome Research, 10 (3): 1266-80.

Sheikh, H.; Siddiqui, M.; Uddin, S.M.M.; Haq, A. and Yaqoob, U. (2018): "The Clinicopathological Profile of Eosinophilic Myocarditis". Cureus, 10 (12): e3677.

Tambourgi, D.V.; Dos Santos, M.C.; Furtado, M.de.F.; De Freitas, M.C.; Da Silva, W.D. and Kipnis, T.L. (1994): "Pro-inflammatory activities in elapid snake venoms". British Journal of Pharmacology, 112(3): 723-727.

Tan, N.H. and Armugam,A. (1990): "In Vivo Interactions between Neurotoxin, Cardiotoxin and Phospholipases A2 Isolated from Malayan Cobra (Naja Naja Sputatrix) Venom”. Toxicon, 28 (10): 1193-98.

Thygesen, K.; Alpert, J.S.; Jaffe, A.S.; Simoons, M.L.; Chaitman, B.R.; White, H.D.; on behalf of the Joint: ESC/ACCF/AHA/WHF Task Force for the Universal Definition of Myocardial Infarction: Thygesen, K.; Alpert, J.S.; White, H.D.; Jaffe, A.S.; Katus, H.A.; Apple, F.S.; Lindahl, B.; Morrow, D.A.; Chaitman, B.A.; Clemmensen, P.M.; Johanson, P. et al. and ESC. Committee for Practice Guidelines (CPG) (2012): "Third universal definition of myocardial infarction". European heart journal, 33(20): 2551-2567.

Wang, C.H. and Wu, W.g. (2005): "Amphiphilic $\beta$ Sheet Cobra Cardiotoxin Targets Mitochondria and Disrupts Its Network." FEBS Letters, 579 (14): 3169-74.

Wang, C.H.; Liu, J.H.; Lee, S.C.; Hsiao, C.D. and Wu, W. G. (2006): "Glycosphingolipid-facilitated membrane insertion and internalization of cobra cardiotoxin: The sulfatide cardiotoxin complex structure in a membrane-like environment suggests a lipid-dependent cell-penetrating mechanism for membrane binding polypeptides". Journal of Biology and Chemistry, 281(1): 656-667.

Wu, M.; Ming, W.; Tang, Y.; Zhou, S.; Kong, T. and Dong, W. (2013): "The Anticancer Effect of 
Cytotoxin 1 from Naja Atra Cantor Venom Is Mediated by a Lysosomal Cell Death Pathway Involving Lysosomal Membrane Permeabilization and Cathepsin B Release". The American Journal of Chinese Medicine, 41 (3): 643-63.
Yeh, E.T.H.; Tong, A.T.; Lenihan, D.J.; Yusuf,S.W.; Swafford, J.; Champion, C.; Durand, J.B.; Gibbs, H.; Zafarmand, A.A. and Ewer, M.S. (2004): "Cardiovascular Complications of Cancer Therapy: Diagnosis, Pathogenesis, and Management”. Circulation, 109 (25): 3122-31. 